\title{
RATIOS OF PERIODS FOR TENSOR PRODUCT MOTIVES
}

\author{
Chandrasheel Bhagwat and A. Raghuram
}

\begin{abstract}
In this paper, we prove some period relations for the ratio of Deligne's periods for certain tensor product motives. These period relations give a motivic interpretation for certain algebraicity results for ratios of successive critical values for Rankin-Selberg $L$-functions for $\mathrm{GL}_{n} \times \mathrm{GL}_{n^{\prime}}$ proved by Günter Harder and the second author.
\end{abstract}

\section{Introduction and motivation}

1.1. A classical example. To motivate the period relations proved in this paper let us recall a classical theorem due to Shimura [Shi77] on the critical values of $L$-functions attached to modular forms. Let $\varphi=\sum a_{n} q^{n}$ be a primitive holomorphic cusp form of weight $k$ for $\Gamma_{0}(N)$. For a Dirichlet character $\chi$, let $L_{f}(s, \varphi, \chi)=\sum_{n} a_{n} \chi(n) / n^{s}$. There exist $u^{ \pm}(\varphi) \in \mathbb{C}^{\times}$(the periods of $\varphi$ ) such that for any integer $m$ with $1 \leq m \leq$ $k-1$, we have

$$
L_{f}(m, \varphi, \chi) \sim(2 \pi i)^{m} \gamma(\chi) u^{ \pm}(\varphi),
$$

where $\chi(-1)= \pm(-1)^{m}, \gamma(\chi)$ is the Gauß sum of $\chi$, and $\sim$ means equality up to an element of the number field $\mathbb{Q}(\varphi, \chi):=\mathbb{Q}\left(\left\{a_{n}\right\} \cup\{\right.$ values of $\left.\chi\}\right)$. Now suppose $1 \leq m \leq k-2$, then

$$
\frac{L_{f}(m, \varphi, \chi)}{L_{f}(m+1, \varphi, \chi)} \sim(2 \pi i)^{-1} \frac{u^{ \pm}(\varphi)}{u^{\mp}(\varphi)},
$$

assuming the denominator of the left-hand side is nonzero. The $2 \pi$ on the right hand side can be thought of as coming from the $L$-factors at infinity, and if we define $\Omega(\varphi):=\frac{1}{i} \frac{u^{+}(\varphi)}{u^{-}(\varphi)}$, then the ratio of successive critical values of the completed $L$-function looks like:

$$
\frac{L(m, \varphi, \chi)}{L(m+1, \varphi, \chi)} \sim \Omega(\varphi)^{\chi(-1)(-1)^{m}} .
$$

Such an algebraicity result for ratios of successive critical values has been generalized by Günter Harder; see [Hard10] and references therein to his earlier papers. This was further generalized by Harder and the second author [HaRa11] which we now briefly recall. 
1.2. A generalization. Let $\pi$ (resp., $\pi^{\prime}$ ) be a cohomological cuspidal automorphic representation of $\mathrm{GL}_{n}(\mathbb{A})$ (resp., $\mathrm{GL}_{n^{\prime}}(\mathbb{A})$ ), where $\mathbb{A}$ is the ring of adèles of $\mathbb{Q}$. Implicit in this data is a pure dominant integral highest weight $\lambda$ (resp., $\lambda^{\prime}$ ) for the algebraic group $\mathrm{GL}_{n} / \mathbb{Q}$ (resp., $\mathrm{GL}_{n^{\prime}} / \mathbb{Q}$ ). Assume that $n$ is even and $n^{\prime}$ is odd. Let $\mathbb{E}$ be a number field containing the rationality fields $\mathbb{Q}(\pi)$ and $\mathbb{Q}\left(\pi^{\prime}\right)$. To the representation $\pi$ and to any embedding $\iota: E \rightarrow \mathbb{C}$, there exist certain relative periods $\Omega(\pi, \iota) \in \mathbb{C}^{\times}$and the collection of these periods, as $\iota$ varies, is well-defined up to $\mathbb{E}^{\times}$. (See [HaRa11].) One may say that one has attached $\Omega(\pi) \in\left(\mathbb{E} \otimes_{\mathbb{Q}} \mathbb{C}\right)^{\times} / \mathbb{E}^{\times}$. Suppose that $m \in \frac{1}{2}+\mathbb{Z}$ is such that both $m$ and $m+1$ are critical for the Rankin-Selberg $L$-function $L\left(s, \pi \times \pi^{\prime}\right)$. Then under a certain assumption involving only $\lambda$ and $\lambda^{\prime}$ (called the combinatorial lemma in loc.cit.), by studying rank one Eisenstein cohomology of $\mathrm{GL}_{n+n^{\prime}}$, it has been shown that

$$
\frac{L\left(m, \pi \times \pi^{\prime}\right)}{L\left(m+1, \pi \times \pi^{\prime}\right)} \sim \Omega(\pi)^{\epsilon_{\pi^{\prime}} \epsilon_{m}} c\left(\pi_{\infty}, \pi_{\infty}^{\prime}\right),
$$

where $\sim$ means equality in $\left(\mathbb{E} \otimes_{\mathbb{Q}} \mathbb{C}\right)^{\times} / \mathbb{E}^{\times} ; \epsilon_{\pi^{\prime}}$ is a sign depending only on $\pi^{\prime} ; \epsilon_{m}$ depends only on the parity of the integer $m-\frac{1}{2} ; c\left(\pi_{\infty}, \pi_{\infty}^{\prime}\right)$ is a nonzero complex number depending only on the representations at infinity (it is expected that this number is rational). Note a piquant feature: it seems that the representation $\pi$ has a bigger role to play in the right-hand side, and that $\pi^{\prime}$ contributes only a sign in the exponent of $\Omega(\pi)$.

1.3. Motivic interpretation. Every known algebraicity statement on critical values of $L$-functions comes under the umbrella of a celebrated conjecture of Deligne [Del79] on critical values of motivic $L$-functions. The purpose of this paper is to look at (1.1) and (1.2) from the perspective of Deligne's conjecture. Let $M$ be a critical motive over $\mathbb{Q}$ with coefficients in a field $\mathbb{E}$. Then Deligne attaches two periods $c^{ \pm}(M) \in\left(\mathbb{E} \otimes_{\mathbb{Q}} \mathbb{C}\right)^{\times} / \mathbb{E}^{\times}$to $M$ by comparing the Betti and de Rham realizations of $M$. The finite part of the $\mathbb{E} \otimes_{\mathbb{Q}} \mathbb{C}$-valued $L$-function $L_{f}(s, M)$ is defined in terms of the $\ell$-adic realization of $M$. Suppose $s=0$ is critical for the $L$-function then Deligne predicts that $L_{f}(0, M) \sim c^{+}(M)$. Further, if $s=1$ is also critical then $L_{f}(1, M) \sim(2 \pi i)^{d^{-}(M)} c^{-}(M)$ for a certain $d^{-}(M) \in \mathbb{Z}$. Hence, the ratio $L_{f}(0, M) / L_{f}(1, M) \sim(2 \pi i)^{-d^{-}(M)} c^{+}(M) / c^{-}(M)$. As in the case of modular forms, the power of $2 \pi$ can be interpreted as a relevant ratio of $L$-factors from infinity (which depend only on the Hodge types of $M)$. Hence, if we wish to consider a ratio of successive critical values then we need to consider the ratio of periods $c^{+}(M) / c^{-}(M)$. To see (1.2), assume that $M$ is pure and of even rank, and consider another pure motive $M^{\prime}$ whose rank is odd. Assume that $M \otimes M^{\prime}$ is critical. Further, assume that all the nonzero Hodge numbers of $M$ and $M^{\prime}$ are 1 . One of the main results of this paper (Theorem 3.1.1) states that in $\left(\mathbb{E} \otimes_{\mathbb{Q}} \mathbb{C}\right)^{\times} / \mathbb{E}^{\times}$we have

$$
\frac{c^{+}\left(M \otimes M^{\prime}\right)}{c^{-}\left(M \otimes M^{\prime}\right)}=\left(\frac{c^{+}(M)}{c^{-}(M)}\right)^{\epsilon\left(M^{\prime}\right)},
$$

where $\epsilon\left(M^{\prime}\right)$ is the sign by which complex conjugation acts on the middle Hodge type of $M^{\prime}$.

The proof of these period relations is based on the formalism of Yoshida [Yos01] on periods of tensor product motives, which need not only $c^{ \pm}(M)$ but also other 
invariants attached to $M$. In Section 2, we recall the relevant parts of his paper that we need. Sections 3 and 4 are the two main sections of this paper.

In Section 5, we prove a couple of variations of (1.3) when the ranks of both the motives have the same parities. We start with the easy case when both $M$ and $M^{\prime}$ have even rank; in this situation we have the following period relation in $\left(\mathbb{E} \otimes_{\mathbb{Q}} \mathbb{C}\right)^{\times} / \mathbb{E}^{\times}$:

$$
c^{+}\left(M \otimes M^{\prime}\right)=c^{-}\left(M \otimes M^{\prime}\right) .
$$

The relations (1.3) and (1.4) are combined together in Theorem 5.2.1 where we present a period relation when $M$ has even rank, and $M^{\prime}$ is a direct sum of critical motives. Next, we consider the somewhat more difficult case when both $M$ and $M^{\prime}$ have odd rank; in this situation we have the following equality in $\left(\mathbb{E} \otimes_{\mathbb{Q}} \mathbb{C}\right)^{\times} / \mathbb{E}^{\times}$:

$$
\frac{c^{+}\left(M \otimes M^{\prime}\right)}{c^{-}\left(M \otimes M^{\prime}\right)}=\left(\frac{c^{+}(M)}{c^{-}(M)}\right)^{\epsilon\left(M^{\prime}\right)}\left(\frac{c^{+}\left(M^{\prime}\right)}{c^{-}\left(M^{\prime}\right)}\right)^{\epsilon(M)}
$$

See Theorem 5.3.2.

There has been a long tradition, since the foundational paper by Deligne [Del79], on period relations for motivic periods and what such relations say about the special values of automorphic $L$-functions; see, for example, Blasius [Bla87], Harris [Harr97], Panchishkin [Pan94], and Yoshida [Yos01]. The reader should view this paper from the perspective of such a tradition.

\section{Special polynomials, motivic periods and results of Yoshida}

In this section, we begin by briefly reviewing the notion of a critical motive. Then we review some results of Yoshida [Yos01] that will be useful for our proofs.

2.1. Critical motives. Let $M$ be a pure motive defined over $\mathbb{Q}$ with coefficients in a number field $\mathbb{E}$. Every pure motive over $\mathbb{Q}$ conjecturally arises, up to a Tate twist, as a submotive of the cohomology motive $H^{*}(X)$ of an algebraic variety $X$ over $\mathbb{Q}$. In this paper, we consider the motives in the sense of their Betti, de Rham and $\ell$-adic realizations as in Deligne [Del79].

Let $H_{\mathrm{B}}(M)$ be the Betti realization of $M$. It is a finite-dimensional vector space over $\mathbb{E}$. The rank $d(M)$ of $M$ is defined to be $\operatorname{dim}_{\mathbb{E}}\left(H_{\mathrm{B}}(M)\right)$. Write

$$
H_{\mathrm{B}}(M)=H_{\mathrm{B}}^{+}(M) \oplus H_{\mathrm{B}}^{-}(M),
$$

where $H_{\mathrm{B}}^{ \pm}(M)$ are the \pm 1-eigenspaces for the action of complex conjugation $\rho$ on $H_{\mathrm{B}}(M)$. Let $d^{ \pm}(M)$ be the $\mathbb{E}$-dimension of $H_{\mathrm{B}}^{ \pm}(M)$. The Betti realization has a Hodge decomposition:

$$
H_{\mathrm{B}}(M) \otimes_{\mathbb{Q}} \mathbb{C}=\bigoplus_{p, q \in \mathbb{Z}} H^{p, q}(M),
$$

where $H^{p, q}(M)$ is a free $\mathbb{E} \otimes \mathbb{C}$-module of rank $h_{M}^{p, q}$. The numbers $h_{M}^{p, q}$ are called the Hodge numbers of $M$. Purity of $M$ means that there is an integer $w$ such that $H^{p, q}(M)=\{0\}$ if $p+q \neq w$. Henceforth, we assume that all our motives are pure. The number $w$ is called the weight of $M$. We also have $\rho\left(H^{p, q}(M)\right)=H^{q, p}(M)$; and hence $\rho$ acts on the (possibly zero) middle Hodge type $H^{w / 2, w / 2}(M)$. 
Let $H_{\mathrm{DR}}(M)$ be the de Rham realization of $M$; it is a $d(M)$-dimensional vector space over $\mathbb{E}$. There is a comparison isomorphism of $\mathbb{E} \otimes_{\mathbb{Q}} \mathbb{C}$-modules:

$$
I: H_{\mathrm{B}}(M) \otimes_{\mathbb{Q}} \mathbb{C} \longrightarrow H_{\mathrm{DR}}(M) \otimes_{\mathbb{Q}} \mathbb{C} .
$$

The de Rham realization has a Hodge filtration $F^{p}(M)$ which is a decreasing filtration of $\mathbb{E}$-subspaces of $H_{\mathrm{DR}}(M)$ such that

$$
I\left(\bigoplus_{p^{\prime} \geq p} H^{p^{\prime}, q}(M)\right)=F^{p}(M) \otimes_{\mathbb{Q}} \mathbb{C} .
$$

Write the Hodge filtration as

$$
H_{\mathrm{DR}}(M)=F^{p_{1}}(M) \supset F^{p_{2}}(M) \supset \cdots \supset F^{p_{m}}(M) \supset F^{p_{m}+1}(M)=\{0\} ;
$$

all the inclusions are proper and there are no other filtration-pieces between two successive members. The numbers $p_{\mu}$ are such that, $h_{M}^{p_{\mu}, w-p_{\mu}} \neq 0$. We assume that the numbers $p_{\mu}$ are maximal among all the choices. Let $s_{\mu}=h_{M}^{p_{\mu}, w-p_{\mu}}$ for $1 \leq \mu \leq m$. Purity plus the action of complex conjugation on Hodge types says that the numbers $p_{j}$ and $s_{\mu}$ satisfy $p_{j}+p_{m+1-j}=w, \forall 1 \leq j \leq m$, and $s_{\mu}=s_{m+1-\mu}, \forall 1 \leq \mu \leq m$.

We say that the motive $M$ is critical if there exist $\mathfrak{p}^{+}, \mathfrak{p}^{-} \in \mathbb{Z}$ such that

$$
\sum_{i=1}^{\mathfrak{p}^{+}} s_{i}=d^{+}(M), \quad \sum_{i=1}^{\mathfrak{p}^{-}} s_{i}=d^{-}(M) .
$$

In this case, one says that $F^{ \pm}(M)$ exists and equals $F^{\mathfrak{p}^{ \pm}}(M)$. It is easy to see that $M$ is critical if and only if complex conjugation acts by a scalar on the middle Hodge type (provided the middle Hodge type exists); in this situation we denote this scalar by $\epsilon(M)$.

2.2. Period invariants. The period matrix of $M$ is defined in terms of $\mathbb{E}$-bases for the spaces $H_{\mathrm{B}}^{ \pm}(M)$ and $H_{\mathrm{DR}}(M)$. Let $\left\{v_{1}, v_{2}, \ldots, v_{d^{+}(M)}\right\}$ be an $\mathbb{E}$-basis of $H_{\mathrm{B}}^{+}(M)$, and similarly, $\left\{v_{d^{+}(M)+1}, v_{d^{+}(M)+2}, \ldots, v_{d(M)}\right\}$ be an $\mathbb{E}$-basis of $H_{\mathrm{B}}^{-}(M)$. For the de Rham realization, let $\left\{w_{1}, w_{2}, \ldots, w_{d(M)}\right\}$ be a basis of $H_{\mathrm{DR}}(M)$ over $\mathbb{E}$ such that $\left\{w_{s_{1}+s_{2}+\cdots+s_{\mu-1}+1}, \ldots, w_{d(M)}\right\}$ is a basis of $F^{p_{\mu}}(M)$ for $1 \leq \mu \leq m$. The period matrix $X$ of $M$ is the matrix which represents the comparison isomorphism between the two realizations of $M$ with respect to the bases chosen above.

Let $\mathbb{F}$ be a number field. Suppose $d$ is a positive integer. Fix a partition $s_{1}+s_{2}+$ $\cdots+s_{m}=d$. Let $P_{m}$ be the corresponding lower parabolic subgroup of $\operatorname{GL}(d, F)$. Given an $m$-tuple of integers $\left(a_{i}\right)_{1 \leq i \leq m}$, define an algebraic character $\lambda_{1}$ of $P_{m}$ by

$$
\lambda_{1}\left(\left(\begin{array}{cccc}
p_{11} & 0 & \ldots & 0 \\
* & p_{22} & \ldots & 0 \\
* & * & \ddots & \ldots \\
* & * & * & p_{m m}
\end{array}\right)\right)=\prod_{1 \leq i \leq m}\left(\operatorname{det} p_{i i}\right)^{a_{i}} ; \quad p_{\mathrm{ii}} \in \operatorname{GL}\left(s_{i}\right)
$$

Let $d=d^{+}+d^{-}$. Given $k^{+}, k^{-} \in \mathbb{Z}$, define a character $\lambda_{2}$ of $\operatorname{GL}\left(d^{+}\right) \times \operatorname{GL}\left(d^{-}\right)$by

$$
\lambda_{2}\left(\left(\begin{array}{ll}
a & 0 \\
0 & b
\end{array}\right)\right)=(\operatorname{det} a)^{k^{+}}(\operatorname{det} b)^{k^{-}}, \quad a \in \operatorname{GL}\left(d^{+}\right), b \in \operatorname{GL}\left(d^{-}\right) .
$$


Let $f(x)$ be a polynomial with rational coefficients which satisfies the following equivariance condition with respect to the left action of $P_{m}$ and the right action of $\mathrm{GL}\left(d^{+}\right) \times \mathrm{GL}\left(d^{-}\right)$on the matrix ring $M_{d}(F)$ :

$$
f(p x \gamma)=\lambda_{1}(p) f(x) \lambda_{2}(\gamma), \quad \forall p \in P_{m}, \quad \forall \gamma \in \mathrm{GL}\left(d^{+}\right) \times \mathrm{GL}\left(d^{-}\right) .
$$

A polynomial is said to have admissibility type $\left\{\left(a_{1}, a_{2}, \ldots, a_{m}\right),\left(k^{+}, k^{-}\right)\right\}$if it satisfies (2.3). Yoshida [Yos01, Theorem 1] proves that the space of polynomials of a given admissibility type is atmost one.

Lemma 2.2.2. Suppose $f(x)$ has admissibility type $\left\{\left(a_{1}, a_{2}, \ldots, a_{m}\right),\left(k_{1}^{+}, k_{1}^{-}\right)\right\}$, and $g(x)$ has admissibility type $\left\{\left(b_{1}, b_{2}, \ldots, b_{m}\right),\left(k_{2}^{+}, k_{2}^{-}\right)\right\}$, then $h(x)=f(x) g(x)$ has admissible type:

$$
\left\{\left(a_{1}+b_{1}, a_{2}+b_{2}, \ldots, a_{m}+b_{m}\right),\left(k_{1}^{+}+k_{2}^{+}, k_{1}^{-}+k_{2}^{-}\right)\right\} .
$$

Proof. Follows from (2.3).

Example 2.2.3. Let $f(x)=\operatorname{det}(x)$ for a matrix $x \in M_{d}(F)$. Then $f(x)$ is of admissibility type $\{(1,1,1, \ldots, 1),(1,1)\}$.

Let $f^{ \pm}(x)$ be the upper left (resp., upper right) $d^{ \pm} \times d^{ \pm}$determinant of $x$. Then it can be seen that the admissibility types of $f^{+}(x)$ and $f^{-}(x)$ are respectively given by

$$
\begin{aligned}
& \left\{(\underbrace{1,1,1, \ldots, 1}_{\mathfrak{p}^{+}}, 0,0, \ldots, 0),(1,0)\right\}, \\
& \left\{(\underbrace{1,1,1, \ldots, 1}_{\mathfrak{p}^{-}}, 0,0, \ldots, 0),(0,1)\right\} .
\end{aligned}
$$

Yoshida interprets the period invariants of the period matrix $X$ via some special polynomials as $\delta(M)=f(X)$ and $c^{ \pm}(M)=f^{ \pm}(X)$. The values in $(\mathbb{E} \otimes \mathbb{C})^{\times}$of these polynomials on a period matrix defines an element of $(\mathbb{E} \otimes \mathbb{C})^{\times} / \mathbb{E}^{\times}$, which is independent of the de Rham and Betti bases chosen to define the period matrix.

2.3. Tensor product motives. The category of motives over $\mathbb{Q}$ with coefficients in $\mathbb{E}$ admits a tensor product. The realizations for the tensor product are naturally identified with the tensor products of the realizations. Yoshida [Yos01, Proposition 12] describes the admissibility types of the polynomials, which correspond to the periods $c^{ \pm}\left(M \otimes M^{\prime}\right)$ of the tensor product motive $M \otimes M^{\prime}$.

Let $X$ and $Y$ be the period matrices of the motives $M$ and $M^{\prime}$, respectively. Let $R=\mathbb{E} \otimes_{\mathbb{Q}} \mathbb{C}$. Suppose $d(M)$ and $d\left(M^{\prime}\right)$ are the ranks of $M$ and $M^{\prime}$, respectively. The numbers $d^{ \pm}\left(M^{\prime}\right)$ are the dimensions of the \pm 1 -eigenspaces for $H_{\mathrm{B}}\left(M^{\prime}\right)$. Write $X$ and $Y$ in the following way:

$$
X=\left(\begin{array}{cc}
X_{1}^{+} & X_{1}^{-} \\
X_{2}^{+} & X_{2}^{-} \\
\vdots & \vdots \\
X_{d(M)}^{+} & X_{d(M)}^{-}
\end{array}\right), \quad Y=\left(\begin{array}{cc}
Y_{1}^{+} & Y_{1}^{-} \\
Y_{2}^{+} & Y_{2}^{-} \\
\vdots & \vdots \\
Y_{d\left(M^{\prime}\right)}^{+} & Y_{d\left(M^{\prime}\right)}^{-}
\end{array}\right),
$$

where $X_{i}^{ \pm} \in R^{d^{ \pm}(M)}$ and $Y_{l}^{ \pm} \in R^{d^{ \pm}\left(M^{\prime}\right)}$. 
Given $1 \leq i \leq d(M)$, let $1 \leq \mu \leq m$ be such that

$$
s_{1}+s_{2}+\cdots s_{\mu-1}<i \leq s_{1}+s_{2}+\cdots+s_{\mu} .
$$

The Hodge level $w\left(X_{i}\right)^{ \pm}$is defined to be the integer $p_{\mu}$ (cf. (2.2)). The Hodge level $w\left(Y_{l}\right)^{ \pm}$is defined analogously. Suppose the motive $M \otimes M^{\prime}$ is critical. Consider the Hodge filtrations of the motives $M, M^{\prime}$ and $M \otimes M^{\prime}$.

$$
\begin{gathered}
H_{d R}(M)=F^{i_{1}}(M) \supset F^{i_{2}}(M) \supset \cdots \supset F^{i_{m_{1}}}(M) \supset(0), \\
H_{d R}\left(M^{\prime}\right)=F^{j_{1}}\left(M^{\prime}\right) \supset F^{j_{2}}\left(M^{\prime}\right) \supset \cdots \supset F^{j_{m_{2}}}\left(M^{\prime}\right) \supset(0), \\
H_{d R}\left(M \otimes M^{\prime}\right)=F^{k_{1}}\left(M \otimes M^{\prime}\right) \supset F^{k_{2}}\left(M \otimes M^{\prime}\right) \supset \cdots \supset F^{k_{m}}\left(M \otimes M^{\prime}\right) \supset(0) .
\end{gathered}
$$

Let $u_{i}$ denote the Hodge numbers of $M \otimes M^{\prime}$. Hence there exist integers $q^{+}, q^{-}$such that

$$
u_{1}+u_{2}+\cdots+u_{q^{ \pm}}=d^{ \pm}\left(M \otimes M^{\prime}\right) .
$$

The integers $a_{\mu}^{ \pm}$are defined by

$$
a_{\mu}^{ \pm}=\left|\left\{l: 1 \leq l \leq d\left(M^{\prime}\right), p_{\mu}+w\left(Y_{l}\right)<k_{q^{ \pm}}\right\}\right| .
$$

From the definition of the period, it follows that $c^{+}\left(M \otimes M^{\prime}\right)$ is the determinant of the square matrix $Z^{+}$of size $d^{+}(M) d^{+}\left(M^{\prime}\right)+d^{-}(M) d^{-}\left(M^{\prime}\right)$ defined by

$$
Z^{+}=\left(X_{i}^{+} \otimes Y_{l}^{+}, X_{i}^{-} \otimes Y_{l}^{-}: w\left(X_{i}^{ \pm}\right)+w\left(Y_{l}^{ \pm}\right)<k_{q^{+}}\right) \text {. }
$$

Similarly, one observes that $c^{-}\left(M \otimes M^{\prime}\right)$ is the determinant of the square matrix $Z^{-}$of size $d^{+}(M) d^{-}\left(M^{\prime}\right)+d^{-}(M) d^{+}\left(M^{\prime}\right)$ defined by

$$
Z^{-}=\left(X_{i}^{+} \otimes Y_{l}^{-}, X_{i}^{-} \otimes Y_{l}^{+}: w\left(X_{i}^{ \pm}\right)+w\left(Y_{l}^{ \pm}\right)<k_{q^{-}}\right) .
$$

The determinants of $Z^{ \pm}$can be expressed in the form $h^{ \pm}(X, Y)$, where $h^{ \pm}(x, y)$ are polynomial functions. For a fixed $y$, the function $h^{ \pm}(x, y)$ has admissibility type

$$
\left\{\left(a_{\mu}^{ \pm}: 1 \leq \mu \leq m,\left(d^{ \pm}\left(M^{\prime}\right), d^{\mp}\left(M^{\prime}\right)\right)\right\} .\right.
$$

Define the integers $\left(a^{*}\right)_{\nu}^{ \pm}$for the motive $M^{\prime}$ analogous to $(2.5)$ above. It follows that the data $\left(\left\{\left(a^{*}\right)_{\nu}^{ \pm}\right\}\right),\left(d^{ \pm}(M), d^{\mp}(M)\right)$ describes the admissibility type of $h^{ \pm}(x, y)$ for a fixed $x$.

From the uniqueness property [Yos01, Theorem 1], it follows that the polynomials $h^{ \pm}(x, y)$ can be expressed as $h^{ \pm}(x, y)=\phi^{ \pm}(x) \psi^{ \pm}(y)$, where $\phi^{ \pm}(x)$ and $\psi^{ \pm}(y)$ are polynomials with the following admissibility types, respectively:

$$
\begin{gathered}
\left\{a_{\mu}^{ \pm}:\left(d^{ \pm}\left(M^{\prime}\right), d^{\mp}\left(M^{\prime}\right)\right)\right\}, \\
\left\{\left(a^{*}\right)_{\nu}^{ \pm}:\left(d^{ \pm}(M), d^{\mp}(M)\right)\right\} .
\end{gathered}
$$

\section{Period relations for motives over $\mathbb{Q}$}

3.1. We now state and prove one of the main results of this paper.

Theorem 3.1.1. Let $M$ and $M^{\prime}$ be pure motives over $\mathbb{Q}$ with coefficients in a number field $\mathbb{E}$. Suppose they satisfy the following properties:

(1) $\operatorname{rank}(M)=d(M)$ is even, and $\operatorname{rank}\left(M^{\prime}\right)=d\left(M^{\prime}\right)$ is odd.

(2) All the nonzero Hodge numbers $h_{M}^{p, q}$ and $h_{M^{\prime}}^{p, q}$ are equal to 1.

(3) The tensor product motive $M \otimes M^{\prime}$ is critical. 
Hypothesis (1) and (2) imply that $M$ and $M^{\prime}$ are critical, and furthermore that complex conjugation acts as a scalar, denoted $\epsilon\left(M^{\prime}\right)$, on the one-dimensional middle Hodge type of $M^{\prime}$. Then the periods $c^{ \pm}(M)$ and $c^{ \pm}\left(M \otimes M^{\prime}\right)$ are related by the following equation in $\left(\mathbb{E} \otimes_{\mathbb{Q}} \mathbb{C}\right)^{\times} / \mathbb{E}^{\times}$:

$$
\frac{c^{+}\left(M \otimes M^{\prime}\right)}{c^{-}\left(M \otimes M^{\prime}\right)}=\left(\frac{c^{+}(M)}{c^{-}(M)}\right)^{\epsilon\left(M^{\prime}\right)} .
$$

3.2. Proof of Theorem 3.1.1. The condition on Hodge numbers of $M$ and $M^{\prime}$ guarantees that the motives $M$ and $M^{\prime}$ are critical, and $d^{+}(M)=d^{-}(M)=d(M) / 2$ and $d^{+}\left(M^{\prime}\right)=d^{-}\left(M^{\prime}\right) \pm 1$; indeed, $d^{+}\left(M^{\prime}\right)=d^{-}\left(M^{\prime}\right)+\epsilon\left(M^{\prime}\right)$. Consider the motive $M \otimes M^{\prime}$. Since it is critical and of even rank, it follows that $d^{ \pm}\left(M \otimes M^{\prime}\right)=$ $d(M) d\left(M^{\prime}\right) / 2$.

Let $X$ and $Y$ be the period matrices of $M$ and $M^{\prime}$, resp. The period $c^{ \pm}\left(M \otimes M^{\prime}\right)$ is given by a polynomial $h^{ \pm}(X, Y)$. Here $h^{ \pm}(x, y)=\phi^{ \pm}(x) \psi^{ \pm}(y)$ and the polynomials $\phi^{ \pm}(x)$ and $\psi^{ \pm}(y)$ are of certain admissible types. The desired property follows from the analogous relation between the invariant polynomials $\phi^{ \pm}(x), \psi^{ \pm}(y)$ and $f^{ \pm}(x)$. To prove it, we compare their admissibility types under the hypothesis of the theorem.

Since $d^{+}(M)=d^{-}(M)$ and $d^{+}\left(M^{\prime}\right)=d^{-}\left(M^{\prime}\right)+\epsilon\left(M^{\prime}\right)$, we have $\mathfrak{p}_{M}^{+}=\mathfrak{p}_{M}^{-}, \mathfrak{p}_{M^{\prime}}^{+}=$ $\mathfrak{p}_{M^{\prime}}^{-}+\epsilon\left(M^{\prime}\right), d^{+}\left(M \otimes M^{\prime}\right)=d^{-}\left(M \otimes M^{\prime}\right)$ and $q^{+}=q^{-}$. As a result, we have the following relations:

$$
\begin{gathered}
a_{\mu}^{+}=a_{\mu}^{-} \quad \text { for } \quad 1 \leq \mu \leq d(M), \\
\left(a^{*}\right)_{\nu}^{+}=\left(a^{*}\right)_{\nu}^{-} \quad \text { for } \quad 1 \leq \nu \leq d\left(M^{\prime}\right) .
\end{gathered}
$$

From (2.6), (2.7), (3.1) and (3.2), it follows that the admissibility types (and hence the functions themselves in view of their uniqueness) of $\psi^{+}(y)$ and $\psi^{-}(y)$ are equal up to $\mathbb{Q}^{\times}$-multiples, which we write as

$$
\psi^{+}(y) \approx \psi^{-}(y) .
$$

Furthermore, the above conditions also imply that the admissibility types of $\phi^{ \pm}(x)$ and $f^{ \pm}(x)$ are related as we now explain. It is convenient to consider two cases:

Case (i): $\epsilon\left(M^{\prime}\right)=1$.

Here $d^{+}\left(M^{\prime}\right)=d^{-}\left(M^{\prime}\right)+1$. From Lemma 2.2.2, and (2.6), (2.7), (3.1) and (3.2), we see that the admissibility types of $\phi^{+}(x) f^{-}(x)$ and $\phi^{-}(x) f^{+}(x)$ are given respectively by:

$$
\begin{aligned}
& \left\{\left(a_{1}^{+}+1, a_{2}^{+}+1, \ldots a_{d(M) / 2}^{+}+1, a_{1+(d(M) / 2)}^{+}, \ldots, a_{d(M)}^{+}\right),\left(d^{+}\left(M^{\prime}\right), d^{-}\left(M^{\prime}\right)+1\right)\right\}, \\
& \left\{\left(a_{1}^{+}+1, a_{2}^{+}+1, \ldots a_{d(M) / 2}^{+}+1, a_{1+(d(M) / 2)}^{+}, \ldots, a_{d(M)}^{+}\right),\left(d^{-}\left(M^{\prime}\right)+1, d^{+}\left(M^{\prime}\right)\right)\right\},
\end{aligned}
$$

which are identical. Hence, from the uniqueness property of invariant polynomials of a given admissibility type we have

$$
\phi^{+}(x) f^{-}(x) \approx \phi^{-}(x) f^{+}(x), \quad\left(\text { equality up to } \mathbb{Q}^{\times}\right) .
$$

Hence, we get

$$
\phi^{+}(x) \psi^{+}(y) f^{-}(x) \approx \phi^{-}(x) \psi^{-}(y) f^{+}(x)
$$


an equality of polynomials up to $\mathbb{Q}^{\times}$; evaluating on period matrices, we get

$$
c^{+}\left(M \otimes M^{\prime}\right) c^{-}(M) \approx c^{-}\left(M \otimes M^{\prime}\right) c^{+}(M),
$$

which is an equality in $R^{\times}=\left(\mathbb{E} \otimes_{\mathbb{Q}} \mathbb{C}\right)^{\times}$up to $\mathbb{E}^{\times}$. This concludes the proof in case (i).

Case (ii): $\epsilon\left(M^{\prime}\right)=-1$.

Here $d^{+}\left(M^{\prime}\right)=d^{-}\left(M^{\prime}\right)-1$. From an analogous argument as in the previous case we get

$$
\phi^{+}(x) f^{+}(x) \approx \phi^{-}(x) f^{-}(x) .
$$

The rest is similar, and in this case we end up with

$$
c^{+}\left(M \otimes M^{\prime}\right) c^{+}(M) \approx c^{-}\left(M \otimes M^{\prime}\right) c^{-}(M) .
$$

3.3. Our proof of Theorem 3.1.1 relies on the facts that $d^{+}(M)=d^{-}(M)$ and $d^{+}\left(M^{\prime}\right)=d^{-}\left(M^{\prime}\right) \pm 1$. The assumption on the Hodge numbers in Theorem 3.1.1 guarantees these conditions, which in turn are valid for the motives coming from cohomological cuspidal representations. Indeed, Theorem 3.1.1 can be rephrased by the foregoing conditions on the dimensions $d^{ \pm}(M)$ and $d^{ \pm}\left(M^{\prime}\right)$ in the hypotheses.

We may further relax the hypotheses on $M^{\prime}$. Suppose $M^{\prime}$ is a pure critical motive of odd rank. Then, complex conjugation acts by a scalar $\epsilon\left(M^{\prime}\right)$ on the middle Hodge type, and $d^{+}\left(M^{\prime}\right)=d^{-}\left(M^{\prime}\right)+\epsilon\left(M^{\prime}\right) k$, where $k$ is the dimension of this middle Hodge type of $M^{\prime}$. Then the same proof gives:

$$
\frac{c^{+}\left(M \otimes M^{\prime}\right)}{c^{-}\left(M \otimes M^{\prime}\right)}=\left(\frac{c^{+}(M)}{c^{-}(M)}\right)^{\epsilon\left(M^{\prime}\right) k} .
$$

\section{Period relations for motives over totally real fields}

4.1. A factorization result for Deligne's periods over a totally real field. Let $\mathbb{F}$ be a totally real number field. Let $I_{\mathbb{F}}$ be the set of all real embeddings of $\mathbb{F}$ into $\mathbb{C}$. A motive $M$ over $\mathbb{F}$ with coefficients in a number field $\mathbb{E}$ has the following realizations: For each $\sigma \in I_{\mathbb{F}}$ we have a Betti realization of $M$, denoted $H_{\mathrm{B}}(\sigma, M)$, which is a vector space of dimension $d(M)$ over $\mathbb{E}$ together with an action of the complex conjugation which we denote $\rho_{\sigma}$. The de Rham realization of $M$, denoted $H_{\mathrm{DR}}(M)$, is a free $\mathbb{E} \otimes_{\mathbb{Q}} \mathbb{F}$ module of rank $d(M)$ with a decreasing filtration $F_{\mathrm{DR}}^{p}(M)$ of $\mathbb{E} \otimes_{\mathbb{Q}} \mathbb{F}$-modules. For each $\sigma \in I_{\mathbb{F}}$, there is a comparison isomorphism of $\mathbb{E} \otimes_{\mathbb{Q}} \mathbb{C}$-modules

$$
I_{\sigma}: H_{\mathrm{B}}(\sigma, M) \otimes_{\mathbb{Q}} \mathbb{C} \longrightarrow H_{\mathrm{DR}}(M) \otimes_{\mathbb{F}, \sigma} \mathbb{C} .
$$

There is a Hodge decomposition: $H_{\mathrm{B}}(\sigma, M) \otimes_{\mathbb{Q}} \mathbb{C}=\oplus_{p, q} H_{\sigma}^{p, q}(M)$ and $\rho_{\sigma}$ maps $H_{\sigma}^{p, q}(M)$ onto $H_{\sigma}^{q, p}(M)$. The rank of $H_{\sigma}^{p, q}(M)$ is independent of $\sigma$ and is denoted by $h_{M}^{p, q}$; these are the Hodge numbers of $M$.

Suppose $M$ is critical, then for each $\sigma \in I_{\mathbb{F}}$ the periods $c^{ \pm}(\sigma, M)$ are defined in a manner analogous to the case of motives over $\mathbb{Q}$, and for a given $\sigma$, the periods $c^{ \pm}(\sigma, M)$ are well-defined as elements of $(\mathbb{E} \otimes \mathbb{C})^{\times} \bmod (\mathbb{E} \otimes \sigma(\mathbb{F}))^{\times}$. 
Given a motive $M$ over $\mathbb{F}$ with coefficients in $\mathbb{E}$, the restriction of scalars functor gives a motive $R_{\mathbb{F} \mid \mathbb{Q}}(M)$ over $\mathbb{Q}$ with coefficients in $\mathbb{E}$ such that

(1) $H_{\mathrm{DR}}\left(R_{\mathbb{F} \mid \mathbb{Q}}(M)\right)=H_{\mathrm{DR}}(M)$ as an $\mathbb{E}$-vector space of dimension $d(M)[\mathbb{F}: \mathbb{Q}]$, and

(2) $H_{\mathrm{B}}\left(R_{\mathbb{F} \mid \mathbb{Q}}(M)\right)=\bigoplus_{\sigma \in I_{\mathbb{F}}} H_{\mathrm{B}}(\sigma, M)$.

The periods $c^{ \pm}\left(R_{\mathbb{F} \mid \mathbb{Q}}(M)\right)$ have the following factorization:

$$
c^{ \pm}\left(R_{\mathbb{F} \mid \mathbb{Q}}(M)\right)=\left(1 \otimes D_{\mathbb{F}}^{1 / 2}\right)^{d^{ \pm}(M)} \prod_{\sigma \in I_{\mathbb{F}}} c^{ \pm}(\sigma, M) \quad\left(\bmod \mathbb{E}^{\times}\right) .
$$

Here $D_{\mathbb{F}}$ is the absolute discriminant of $\mathbb{F}$. Such a factorization of periods has been observed by many; see, for example, Blasius [Bla97, M.8], Hida [Hid94, p.442] or Panchishkin [Pan94, p.995]. This is closely related to a long history concerning Shimura's conjecture about factorization of periods related to Hilbert modular forms; we refer the reader to Yoshida [Yos95] and Harris [Harr93] and references therein.

4.2. Period relations over totally real fields. An analogue of Theorem 3.1.1 holds for motives over totally real fields under suitably modified hypotheses.

Theorem 4.2.1. Let $M$ and $M^{\prime}$ be motives defined over a totally real number field $\mathbb{F}$ with coefficients in a number field $\mathbb{E}$. Suppose they satisfy the following properties:

(1) $\operatorname{rank}(M)=d(M)$ is even, and $\operatorname{rank}\left(M^{\prime}\right)=d\left(M^{\prime}\right)$ is odd.

(2) All the nonzero Hodge numbers $h_{M}^{p, q}$ and $h_{M^{\prime}}^{p, q}$ are equal to 1.

(3) The motive $M \otimes M^{\prime}$ is critical.

Let $\epsilon\left(\sigma, M^{\prime}\right)$ be the scalar by which the complex conjugation $\rho_{\sigma}$ acts on the rank-one middle Hodge type of $H_{B}\left(\sigma, M^{\prime}\right)$.

(i) For $\sigma \in I_{\mathbb{F}}$, as elements of $(\mathbb{E} \otimes \mathbb{C})^{\times}$we have

$$
\frac{c^{+}\left(\sigma, M \otimes M^{\prime}\right)}{c^{-}\left(\sigma, M \otimes M^{\prime}\right)}=\left(\frac{c^{+}(\sigma, M)}{c^{-}(\sigma, M)}\right)^{\epsilon\left(\sigma, M^{\prime}\right)} \quad\left(\bmod (\mathbb{E} \otimes \sigma(\mathbb{F}))^{\times}\right) .
$$

(ii) As elements of $(\mathbb{E} \otimes \mathbb{C})^{\times}$we have

$$
\frac{c^{+}\left(R_{\mathbb{F} \mid \mathbb{Q}}\left(M \otimes M^{\prime}\right)\right)}{c^{-}\left(R_{\mathbb{F} \mid \mathbb{Q}}\left(M \otimes M^{\prime}\right)\right)}=\prod_{\sigma \in I_{\mathbb{F}}}\left(\frac{c^{+}(\sigma, M)}{c^{-}(\sigma, M)}\right)^{\epsilon\left(\sigma, M^{\prime}\right)} \quad\left(\bmod (\mathbb{E} \otimes \mathcal{F})^{\times}\right),
$$

where $\mathcal{F}$ is any subfield of $\mathbb{C}$ containing $\sigma(\mathbb{F})$ for all $\sigma \in I_{\mathbb{F}}$.

Proof. The proof of Theorem 3.1.1 gives the proof of (i) mutatis mutandis since the discussion involving Yoshida's results (in Section 2.2) works over $\mathbb{Q}$. Next, (i) $\Longrightarrow$ (ii) follows from (4.1); note that the discriminant factor cancels out since $d^{+}\left(M \otimes M^{\prime}\right)=$ $d^{-}\left(M \otimes M^{\prime}\right)=d(M) d\left(M^{\prime}\right) / 2$.

Remark 4.2.2. Panchishkin has conjectured, based on suggestions from Beilinson, that there should exist $\tilde{c}^{ \pm}(\sigma, M) \in(\mathbb{E} \otimes \mathbb{C})^{\times}$well-defined modulo $\mathbb{E}^{\times}$such that for all $\sigma \in I_{\mathbb{F}}$ we have

$$
\tilde{c}^{ \pm}(\sigma, M)=c^{ \pm}(\sigma, M) \quad\left(\bmod (\mathbb{E} \otimes \sigma(F))^{\times}\right) .
$$


(See [Pan94, Conjecture 2.3].) Granting this, statement (ii) of Theorem 4.2.1 can be conjecturally refined as:

$$
\frac{c^{+}\left(R_{\mathbb{F} \mid \mathbb{Q}}\left(M \otimes M^{\prime}\right)\right)}{c^{-}\left(R_{\mathbb{F} \mid \mathbb{Q}}\left(M \otimes M^{\prime}\right)\right)}=\prod_{\sigma \in I_{\mathbb{F}}}\left(\frac{\tilde{c}^{+}(\sigma, M)}{\tilde{c}^{-}(\sigma, M)}\right)^{\epsilon\left(\sigma, M^{\prime}\right)} \quad\left(\bmod \mathbb{E}^{\times}\right) .
$$

\section{Comments on when ranks of $M$ and $M^{\prime}$ have the same parity}

5.1. When the ranks of both $M$ and $M^{\prime}$ are even. In this case, it follows from the hypotheses of Theorem 3.1.1 that $d^{+}(M)=d^{-}(M), d^{+}\left(M^{\prime}\right)=d^{-}\left(M^{\prime}\right)$ and $d^{+}\left(M \otimes M^{\prime}\right)=d^{-}\left(M \otimes M^{\prime}\right)$. Since the tensor product motive $M \otimes M^{\prime}$ is critical by assumption, there exist integers $q^{+}, q^{-}$(as defined in (2.4)) such that

$$
u_{1}+u_{2}+\cdots+u_{q^{ \pm}}=d^{ \pm}\left(M \otimes M^{\prime}\right) .
$$

Thus, we have $q^{+}=q^{-}$and as a result, equations (3.1) and (3.2) are satisfied. From the results of Yoshida ( $c f$. [Yos01, Cor.1, p.1188]) we get

$$
c^{+}\left(M \otimes M^{\prime}\right)=c^{-}\left(M \otimes M^{\prime}\right) .
$$

This ties up very well with known results on critical values. Consider a classical situation: suppose $f$ and $g$ are primitive holomorphic cusp forms of the same level and of weights $k$ and $l$. Suppose $k<l$ and $\psi$ is the nebentypus of $g$. Let us look at the (degree four) Rankin-Selberg $L$-function $L(s, \mathrm{f} \times \mathrm{g})$. Let $l \leq m<k$. Then Shimura [Shi77, Theorem 4] has proved

$$
L_{f}(m, \mathbf{f} \times \mathbf{g}) \sim(2 \pi i)^{2 m-l-1} \gamma(\psi) u^{+}(\mathbf{f}) u^{-}(\mathbf{f}) .
$$

Suppose now that both $m$ and $m+1$ are critical then we get

$$
\frac{L_{f}(m, \mathrm{f} \times \mathrm{g})}{L_{f}(m+1, \mathrm{f} \times \mathrm{g})} \sim(2 \pi i)^{-2} .
$$

As in Section 1.1, we can absorb the $(2 \pi i)^{2}$ into the ratio of $L$-factors at infinity and deduce:

$$
L(m, \mathrm{f} \times \mathrm{g}) \sim L(m+1, \mathrm{f} \times \mathrm{g}) .
$$

It is this statement about $L$-values for $\mathrm{GL}_{2} \times \mathrm{GL}_{2}$ that is motivically interpreted in (5.1) for a tensor product of two rank two motives. The generalization of (5.2) to the context of Rankin-Selberg $L$-functions for $\mathrm{GL}_{n} \times \mathrm{GL}_{n^{\prime}}$, when both $n$ and $n^{\prime}$ are even, is work in progress by Günter Harder and the second author; the results will appear elsewhere.

5.2. Theorem 3.1.1, (3.3) and (5.1) can be combined together as:

Theorem 5.2.1. Let $M$ and $M^{\prime}$ be pure motives over $\mathbb{Q}$ with coefficients in a number field $\mathbb{E}$. Suppose they satisfy the following properties:

(1) $M$ is critical and $d^{+}(M)=d^{-}(M)$.

(2) $M^{\prime}$ is a direct sum of critical motives.

(3) $M \otimes M^{\prime}$ is critical. 
Then the periods $c^{ \pm}(M)$ and $c^{ \pm}\left(M \otimes M^{\prime}\right)$ are related by the following equation in $\left(\mathbb{E} \otimes_{\mathbb{Q}} \mathbb{C}\right)^{\times} / \mathbb{E}^{\times}:$

$$
\frac{c^{+}\left(M \otimes M^{\prime}\right)}{c^{-}\left(M \otimes M^{\prime}\right)}=\left(\frac{c^{+}(M)}{c^{-}(M)}\right)^{\operatorname{Tr}\left(\left.\rho\right|_{H_{\mathrm{B}}\left(M^{\prime}\right)}\right)} .
$$

We leave it to the reader to formulate the analogous statement for motives over a totally real field $\mathbb{F}$ with coefficients in $\mathbb{E}$.

5.3. When the ranks of both $M$ and $M^{\prime}$ are odd. Let $M, M^{\prime}$ be pure motives defined over $\mathbb{Q}$ with coefficients in a number field $\mathbb{E}$. Suppose that both the ranks $d(M)$ and $d\left(M^{\prime}\right)$ are odd. Similar to the earlier even-odd case, assume that all Hodge numbers $h^{p, q}(M), h^{p, q}\left(M^{\prime}\right)$ are less than or equal to one. From this, it follows that $M$ and $M^{\prime}$ are critical and they have nonzero middle Hodge types. Let $\epsilon(M)$ (resp., $\left.\epsilon\left(M^{\prime}\right)\right)$ be the scalar by which complex conjugation acts on the middle Hodge type of $M$ (resp., $M^{\prime}$ ). Suppose the tensor product motive $M \otimes M^{\prime}$ is also critical. It follows that

$$
d^{+}\left(M \otimes M^{\prime}\right)-d^{-}\left(M \otimes M^{\prime}\right)=\epsilon(M) \epsilon\left(M^{\prime}\right) .
$$

Consider the Hodge filtrations on the de Rham realizations of the motives $M, M^{\prime}$ and $M \otimes M^{\prime}$. Let $u_{t}$ be the $t^{t h}$ Hodge number of $M \otimes M^{\prime}$, i.e., the $\mathbb{E}$-dimension of $F^{k_{t}}\left(M \otimes M^{\prime}\right) / F^{k_{t+1}}\left(M \otimes M^{\prime}\right)$. Let $1 \leq r \leq d(M), 1 \leq s \leq d\left(M^{\prime}\right)$. Let $\left\{v_{r}\right\},\left\{w_{s}\right\}$ be $\mathbb{E}$ basis of the one dimensional quotient spaces $F^{i_{r}}(M) / F^{i_{r+1}}(M), F^{j_{s}}\left(M^{\prime}\right) / F^{j_{s+1}}\left(M^{\prime}\right)$ respectively. Then it follows that the set $\left\{v_{r} \otimes w_{s}: i_{r}+j_{s}=k_{t}\right\}$ of classes modulo $F^{k_{t+1}}\left(M \otimes M^{\prime}\right)$ forms a basis of the quotient space $F^{k_{t}}\left(M \otimes M^{\prime}\right) / F^{k_{t+1}}\left(M \otimes M^{\prime}\right)$. The size of this set is $u_{t}$. Since the motive $M \otimes M^{\prime}$ is critical, there exist integers $q^{ \pm}$ such that

$$
d^{ \pm}(M \otimes M)=\sum_{t \leq q^{ \pm}} u_{t}
$$

Thus, $d^{ \pm}(M \otimes M)=\left|\left\{(r, s): 1 \leq r \leq d(M), 1 \leq s \leq d\left(M^{\prime}\right), i_{r}+j_{s} \leq k_{q^{ \pm}}\right\}\right|$. From (5.3) above, we have $q^{+}=q^{-}+\epsilon(M) \epsilon\left(M^{\prime}\right), u_{\max \left(q^{+}, q^{-}\right)}=1$. For $1 \leq r \leq d(M)$, let $a_{r}^{ \pm}=\left|\left\{s: 1 \leq s \leq d\left(M^{\prime}\right), i_{r}+j_{s} \leq k_{q}^{ \pm}\right\}\right|$.

Case $1: q^{+}=q^{-}+1$.

We have

$$
a_{r}^{+}-a_{r}^{-}=\left|\left\{s: 1 \leq s \leq d\left(M^{\prime}\right), k_{q}^{-}<i_{r}+j_{s} \leq k_{q}^{+}\right\}\right| .
$$

Since $u_{q^{+}}=1$, it follows that there exists unique $\left(r_{0}, s_{0}\right)$ such that

- $1 \leq r_{0} \leq d(M), 1 \leq s_{0} \leq d\left(M^{\prime}\right)$.

- $i_{r_{0}}+j_{s_{0}}=k_{q^{+}}$.

- $a_{r_{0}}^{+}-a_{r_{0}}^{-}=1$.

- $a_{r}^{+}=a_{r}^{-}, \quad \forall r \neq r_{0}$.

(In fact $r_{0}=d^{-}(M)+(\epsilon(M)+1) / 2$ and $s_{0}=d^{-}\left(M^{\prime}\right)+\left(\epsilon\left(M^{\prime}\right)+1\right) / 2$.)

Case $2: q^{+}=q^{-}-1$.

We have

$$
a_{r}^{-}-a_{r}^{+}=\left|\left\{s: 1 \leq s \leq d\left(M^{\prime}\right), k_{q}^{+}<i_{r}+j_{s} \leq k_{q}^{-}\right\}\right| .
$$


Since $u_{q^{-}}=1$, it follows that there exists unique $\left(r_{0}, s_{0}\right)$ (same as in Case 1 ) such that

- $1 \leq r_{0} \leq d(M), 1 \leq s_{0} \leq d\left(M^{\prime}\right)$.

- $i_{r_{0}}+j_{s_{0}}=k_{q^{-}}$.

- $a_{r_{0}}^{-}-a_{r_{0}}^{+}=1$.

- $a_{r}^{+}=a_{r}^{-} \quad \forall r \neq r_{0}$.

Define the "dual" data as follows. For $1 \leq s \leq d\left(M^{\prime}\right)$, let

$$
a_{s}^{*, \pm}=\left|\left\{1 \leq r \leq d(M): a_{r}^{ \pm} \geq s\right\}\right| .
$$

It follows (from an argument similar to the case of $a_{r}^{ \pm}$) that, $a_{s_{0}}^{*,+}-a_{s_{0}}^{*,-}=q^{+}-q^{-}$ and $a_{s}^{+}=a_{s}^{-} \quad \forall s \neq s_{0}$.

The following is the analogue of Theorem 3.1.1 in the situation where both motives are of odd rank.

Theorem 5.3.2. Let $M$ and $M^{\prime}$ be pure motives over $\mathbb{Q}$ with coefficients in a number field $\mathbb{E}$. Suppose they satisfy the following properties:

(1) $\operatorname{rank}(M)=d(M)$ and $\operatorname{rank}\left(M^{\prime}\right)=d\left(M^{\prime}\right)$ are odd.

(2) All the nonzero Hodge numbers $h_{M}^{p, q}$ and $h_{M^{\prime}}^{p, q}$ are equal to 1.

(3) The tensor product motive $M \otimes M^{\prime}$ is critical.

Hypothesis (2) implies that the complex conjugation acts as a scalar, denoted $\epsilon(M)$, (resp., $\epsilon\left(M^{\prime}\right)$ ), on the one-dimensional middle Hodge type of $M$ (resp., $\left.M^{\prime}\right)$. Then the periods $c^{ \pm}(M), c^{ \pm}\left(M^{\prime}\right)$ and $c^{ \pm}\left(M \otimes M^{\prime}\right)$ are related by the following equation in $\left(\mathbb{E} \otimes_{\mathbb{Q}} \mathbb{C}\right)^{\times} / \mathbb{E}^{\times}:$

$$
\frac{c^{+}\left(M \otimes M^{\prime}\right)}{c^{-}\left(M \otimes M^{\prime}\right)}=\left(\frac{c^{+}(M)}{c^{-}(M)}\right)^{\epsilon\left(M^{\prime}\right)}\left(\frac{c^{+}\left(M^{\prime}\right)}{c^{-}\left(M^{\prime}\right)}\right)^{\epsilon(M)} .
$$

Proof. From the definition of the Deligne periods, it follows that $c^{+}\left(M \otimes M^{\prime}\right)$ is the determinant of the square matrix $Z^{+}$of size $d^{+}(M) d^{+}\left(M^{\prime}\right)+d^{-}(M) d^{-}\left(M^{\prime}\right)$ defined by

$$
Z^{+}=\left(X_{r}^{+} \otimes Y_{s}^{+}, X_{r}^{-} \otimes Y_{s}^{-}: 1 \leq r \leq d(M), 1 \leq s \leq d\left(M^{\prime}\right): i_{r}+j_{s} \leq k_{q^{+}}\right) .
$$

Similarly, one observes that $c^{-}\left(M \otimes M^{\prime}\right)$ is the determinant of the square matrix $Z^{-}$ of size $d^{+}(M) d^{-}\left(M^{\prime}\right)+d^{-}(M) d^{+}\left(M^{\prime}\right)$ defined by

$$
Z^{-}=\left(X_{r}^{+} \otimes Y_{s}^{-}, X_{r}^{-} \otimes Y_{s}^{+}: 1 \leq r \leq d(M), 1 \leq s \leq d\left(M^{\prime}\right): i_{r}+j_{s} \leq k_{q^{-}}\right) .
$$

Recall that the determinants of $Z^{ \pm}$can be expressed in the form $h^{ \pm}(X, Y)$, where $h^{ \pm}(x, y)$ are polynomial functions. For a fixed $y$, the function $h^{ \pm}(x, y)$ has admissibility type

$$
\left\{\left(a_{\mu}^{ \pm}: 1 \leq \mu \leq d(M)\right),\left(d^{ \pm}\left(M^{\prime}\right), d^{\mp}\left(M^{\prime}\right)\right)\right\} .
$$

The polynomials $h^{ \pm}(x, y)$ can be expressed as $h^{ \pm}(x, y)=\phi^{ \pm}(x) \psi^{ \pm}(y)$ where $\phi^{ \pm}(x)$ and $\psi^{ \pm}(y)$ are polynomials with the following admissibility types respectively $(2.6$, $2.7)$ :

$$
\begin{aligned}
& \left\{\left(a_{\mu}^{ \pm}: 1 \leq \mu \leq d(M)\right), \quad\left(d^{ \pm}\left(M^{\prime}\right), d^{\mp}\left(M^{\prime}\right)\right)\right\}, \\
& \left\{\left(a_{\nu}^{*, \pm}: 1 \leq \nu \leq d\left(M^{\prime}\right)\right), \quad\left(d^{ \pm}(M), d^{\mp}(M)\right)\right\} .
\end{aligned}
$$

The proof follows from the same arguments those in the proof of Theorem 3.1.1. We give the proof for the case when $\epsilon(M)=\epsilon\left(M^{\prime}\right)=+1$ as a sample case. 
Here $q^{+}=q^{-}+1$. So $a_{r}^{+}=a_{r}^{-}$except when $r=d^{+}(M)$ and $a_{d^{+}(M)}^{+}=a_{d^{+}(M)}^{-}+1$. Similarly $a_{s}^{*,+}=a_{s}^{*,-}$ except when $s=d^{+}\left(M^{\prime}\right)$ and $a_{d^{+}\left(M^{\prime}\right)}^{*,}=a_{d^{+}\left(M^{\prime}\right)}^{*,-}+1$. It can be seen that both $\phi^{+} f^{-}$and $\phi^{-} f^{+}$are of same admissibility type given by

$$
\left\{\left(a_{1}^{+}+1, a_{2}^{+}+1, \ldots, a_{d^{-}(M)}^{+}+1, a_{d^{+}(M)}^{+}=a_{d^{+}(M)}^{-}+1, \ldots, a_{d(M)}^{+}\right),\left(d^{+}\left(M^{\prime}\right), d^{+}\left(M^{\prime}\right)\right)\right\} .
$$

On the other hand, $\psi^{+} g^{-}$and $\psi^{-} g^{+}$are of same admissibility type given by $\left\{\left(a_{1}^{*,+}+1, a_{2}^{*,+}+1, \ldots, a_{d^{-}\left(M^{\prime}\right)}^{*,+}+1, a_{d^{+}\left(M^{\prime}\right)}^{*,+}=a_{d^{+}\left(M^{\prime}\right)}^{*,-}+1, \ldots, a_{d\left(M^{\prime}\right)}^{*,+}\right),\left(d^{+}(M), d^{+}(M)\right)\right\}$. The desired relation follows from the uniqueness results in [Yos01].

It is an amusing exercise to use the special values of Dirichlet $L$-functions (see, for example, Neukirch [Neu99, p.442]) and the special values of the symmetric square $L$ functions attached to holomorphic primitive elliptic cusp forms (due to Sturm [Stu80]) to produce examples illustrating Theorem 5.3.2 when $\operatorname{rank}(M)=1$ and $\operatorname{rank}\left(M^{\prime}\right)=$ 1 or 3 .

\section{Acknowledgments}

It is a pleasure to thank Dipendra Prasad who made several suggestions in his attempts to understand the motivic period relations. The comments in Section 3.3 and the formulation of Theorem 5.2.1 were suggested by him. We thank the referee for his/her meticulous comments which helped us enormously in revising the article. C.B. is partially supported by DST-INSPIRE Faculty scheme, award number [IFA- 11MA -05]. A.R. is partially supported by the National Science Foundation (NSF), award number DMS-0856113, and an Alexander von Humboldt Research Fellowship.

\section{References}

[Bla87] D. Blasius, Appendix to Orloff Critical values of certain tensor product L-functions, Invent. Math., 90(1) (1987), 181-188.

[Bla97] D. Blasius, Period relations and critical values of L-functions, Olga Taussky-Todd: in memoriam, Pacific J. Math., (1997), Special Issue, 53-83.

[Del79] P. Deligne, Valeurs de fonctions L et périodes d'intégrales (French), With an appendix by N. Koblitz and A. Ogus. Proc. Symp. Pure Math., XXXIII, Automorphic forms, representations and $L$-functions (Proc. Symp. Pure Math., Oregon State University, Corvallis, Oregon, 1977), Part 2, pp. 313-346, Amer. Math. Soc., Providence, RI, 1979.

[Hard10] G. Harder, Arithmetic aspects of rank one Eisenstein cohomology, Cycles, motives and Shimura varieties, 131-190, Tata Inst. Fund. Res. Stud. Math., Tata Inst. Fund. Res., Mumbai, 2010.

[HaRa11] G. Harder, and A. Raghuram, Eisenstein cohomology and ratios of critical values of Rankin-Selberg L-functions, C. R. Acad. Sci. Paris, Ser. I 349 (2011), 719-724.

[Harr93] M. Harris, L-functions of $2 \times 2$ unitary groups and factorization of periods of Hilbert modular forms, J. AMS, 6(3) (1993), 637-719.

[Harr97] M. Harris, L-functions and periods of polarized regular motives, J. Reine Angew. Math., 483 (1997), 75-161.

[Hid94] H. Hida, On the critical values of L-functions of $G L(2)$ and $G L(2) \times G L(2)$, Duke Math. J., 74(2) (1994), 431-529.

[Neu99] J. Neukirch, Algebraic number theory, Springer-Verlag Berlin Heidelberg, 1999.

[Pan94] A. Panchishkin, Motives over totally real fields and p-adic L-functions, Ann. Inst. Fourier, 44(4) (1994), 989-1023.

[Shi77] G. Shimura, On the periods of modular forms, Math. Ann., 229(3) (1977), 211-221

[Stu80] J. Sturm, Special values of zeta functions, and Eisenstein series of half integral weight, Am. J. Math. 102(2), (1980), 219-240. 
[Yos95] H. Yoshida, On a conjecture of Shimura concerning periods of Hilbert modular forms, Amer. J. Math., 117(4) (1995), 1019-1038.

[Yos01] H. Yoshida, Motives and Siegel modular forms, Amer. J. Math., 123 (2001), 1171-1197.

Indian Institute of Science Education and Research (IISER), Dr. Homi Bhabha Road, Pashan, Pune 411008, India

E-mail address: cbhagwat@iiserpune.ac.in; raghuram@iiserpune.ac.in 